\title{
Enfoque intercultural de salud en la población indígena que vive en territorio colombiano
}

\section{Intercultural approach to health in the indigenous population living in colombian territory}

José Mauricio Hernández-Sarmiento ${ }^{1}$, María Camila Calle-Estrada ${ }^{1}$, María Camila Ospina-Jimenez ${ }^{1}$, Laura Herrera-Almanza ${ }^{1}$, Mabel Dahiana Roldán-Tabares ${ }^{1}$, Lina María Martínez-Sánchez ${ }^{1}$

\section{RESUMEN}

Esta investigación tiene como objetivo principal hacer una reflexión sobre cómo se puede abordar la salud en pueblos indigenas de Colombia, a partir de la experiencia de educación en salud que ha tenido el grupo Piraguas de la Facultad de Medicina de la Universidad Pontificia Bolivariana con la comunidad indigena Emberá. Este es un grupo de proyección social que busca impactar en la salud de las comunidades más vulnerables del país mediante una estrategia de formación en hábitos saludables, propiciando un diálogo de saberes entre la medicina ancestraly la medicina moderna, de manera que

\begin{abstract}
The main objective of this research is to reflect on how health can be addressed in indigenous peoples of Colombia, based on the experience of health education that the Piraguas group of the Faculty of Medicine of the Universidad Pontificia Bolivariana has had with the community Embera indigenous. This is a group of social projection that seeks to impact the health of the most vulnerable communities in the country, through a training strategy in healthy habits, fostering a dialogue of knowledge between ancestral medicine and modern medicine, so that the communities They
\end{abstract}

\section{Historial del artículo:}

Fecha de recepción: 08/06/2020

Fecha de aprobación: 23/05/2021

1 Universidad Pontificia Bolivariana, Escuela de Ciencias de la Salud. Medellín, Colombia.

Correspondencia: Lina María Martínez Sánchez. Universidad Pontificia Bolivariana. Calle 1 \#70-01, Medellín, Colombia. Correo electrónico: linam.martinez@upb.edu.co

Como citar este artículo: Hernández-Sarmiento JM, Calle-Estrada MC, Ospina-Jimenez MC, Herrera-Almanza L, Roldan-Tabares MD, Martínez-Sanchez LM. Enfoque intercultural de salud en la población indígena que vive en territorio colombiano. Revista de la Facultad de Ciencias de la Salud de la Universidad del Cauca. 2021;23(1):23-31. https://doi.org/10.47373/rfcs.2021.v23.1515 
las comunidades puedan ser partícipes de su autocuidado basadas en las herramientas que tienen más accesibles. Este artículo brinda una amplia información sobre el contexto social y cultural de la comunidad Embeń y los métodos por los cuales se puede realizar un enfoque de interculturalidad en la salud en esta población, logrando una interacción simétrica de conocimientos y tradiciones bajo el respeto en busca de la complementariedad.

Palabras clave: Cultura, Educación, Población, Indígena, Salud, Interculturalidad. (DeCS)

\section{INTRODUCCIÓN}

Según Ralph Linton, la cultura es el conjunto de ideas, respuestas emocionales condicionadas y pautas de conducta, que los miembros de una sociedad adquieren mediante educación o imitación, y que comparten en cierto grado (1). Desde este punto de vista, la cultura abarca el conjunto de creencias y valores, instrumentos materiales, forma de legislar y los modelos de comportamiento de los integrantes de una comunidad.

Colombia es un país reconocido por su rica diversidad cultural expresada en la multiplicidad de identidades y expresiones culturales de los pueblos y comunidades que la conforman como Nación (2). Según datos del DANE del ańo 2005 , la población mestiza del país es del 85,9\%, mientras que los grupos étnicos representan el 13,8\%, de los cuales el 10,4\% corresponde a los afrocolombianos y el 3,4\% a los indígenas. Este último grupo corresponde a 1.392 .623 de personas que conforman alrededor de 87 etnias o pueblos indígenas ubicados en los 32 departamentos del país (2). Una de estas etnias es la emberá, la cual cuenta con un total de 37.327 personas auto-reconocidas como pertenecientes a este pueblo indígena y está localizada principalmente en los departamentos del Chocó, Antioquia y Risaralda (3).

Los emberá son denominados lingüísticamente "hombres de río", dado que sus viviendas y cultivos se encuentran a orillas de éste. Este pueblo conserva la lengua Embera, la cual pertenece a la familia lingüística Chocó. Su economía se basa en la agricultura, caza, pesca y la recolección, con una reciente introducción de los hombres al trabajo asalariado. Son considerados como el pueblo indígena con mayor dispersión territorial en Colombia, pero con menor densidad poblacional sobre los territorios que ocupa. La unidad básica de los emberá es la familia, ella tiene poder decisorio sobre sus miembros y allí resuelve sus conflictos a nivel interno (3). can be participants in their self-care based on the tools that are most accessible to them. This article provides extensive information on the social and cultural context of the Embera community and the methods by which an intercultural approach to health can be carried out in this population, achieving a symmetrical interaction of knowledge and traditions under respect in search of complementarity.

Key words: Culture, Education, Population, Indigenous, Health, Interculturality. (MeSH)

Dada esta diversidad cultural, la Constitución Política de Colombia del ańo 1991 al modificar el concepto de nación, reconoce y protege la diversidad étnica y cultural del territorio y establece que es obligación del Estado y de las personas, proteger las riquezas culturales y naturales de la Nación (4). Sin embargo, al igual que muchos países de América Latina, Colombia ha tenido diferentes problemas para reconocer la igualdad de derechos entre los subgrupos del grupo étnico mayoritario del país, los mestizos (5). Por tanto, más difícil aun será lograr reconocer los derechos de los grupos minoritarios, y es prioritario, como lo expresa Pineda Camacho, "que los pueblos indígenas logren un mejor reconocimiento de su autonomía para gobernarse, delimitar sus territorios y auto administrarlos" (6).

Adicional a la falta de reconocimiento por parte del Estado, la mayoría de la población indígena, incluyendo la emberá, se ubica en el área rural del país en los resguardos indígenas legalmente constituidos, en las parcialidades indígenas, o en territorios no delimitados legalmente (2). Así mismo, se observa que el $90 \%$ del territorio perteneciente a los resguardos se encuentra en ocho departamentos de frontera, lo que genera consecuencias económicas y sociales importantes, pues a pesar de la magnitud de la extensión de los resguardos indígenas, el uso del suelo en donde están ubicadas estas comunidades presenta serias restricciones (7). Este aislamiento demográfico de las comunidades, sumado a la gran desigualdad social existente y a los múltiples problemas de orden público, entre ellas confrontaciones armadas y desplazamientos forzados, hace de esta comunidad una población vulnerable, en donde su situación social, económica y política es realmente preocupante.

Por lo anterior, el enfoque intercultural es una opción para que las distintas culturas que viven en el territorio colombiano puedan vivir en paz y desarrollarse, puesto que tanto la paz como la interculturalidad requieren la práctica de valores como tolerancia, respeto, solidaridad, 
justicia y diálogo, entre otros (8). Ambas permiten valorar la diversidad mediante la percepción y el reconocimiento de la valiosa cultura propia y del otro.

La interculturalidad hace alusión, según Walsh, a las relaciones, negociaciones, intercambios culturales e interacciones entre personas, conocimientos, prácticas, racionalidades y principios de vida, que se realizan de manera simétrica, respetando tanto lo propio como lo diferente (9). Del mismo modo, la multiculturalidad consiste en reconocer y proteger la diversidad étnica y cultural, que abarca la convivencia en un mismo territorio de blancos, mestizos, negros e indígenas, con un patrón igualitario en donde todos los grupos comparten los mismos derechos (9).

Es posible aplicar el enfoque intercultural en diversas áreas, una de ellas es la medicina. Dicho enfoque permite evidenciar la armonía y la complementariedad que existe entre sistemas médicos (3), dado que ningún sistema de salud por sí solo es capaz de satisfacer completamente las necesidades de las diferentes comunidades (10). La interculturalidad en salud se entiende como la capacidad de moverse equilibradamente entre conocimientos, creencias y prácticas culturales diferentes respecto a la salud y la enfermedad, la vida y la muerte, el cuerpo biológico, social y relacional (11).

Teniendo en cuenta el concepto de interculturalidad, en el que se plantea un proceso de reciprocidad, en el presente artículo discutimos algunos apartes de la cultura del pueblo emberá referente a la salud, y cómo desde el grupo Piraguas de la Universidad Pontificia Bolivariana proponemos un diálogo respetuoso de saberes en el cual este conocimiento se complemente con la medicina moderna.

\section{PRÁCTICAS Y CONOCIMIENTO DE SALUD EN LAS COMUNIDADES INDÍGENAS}

La medicina tradicional indígena se define como el conjunto de prácticas, enfoques y conocimientos que incorporan una variedad de terapias para mantener el bienestar; tratar, diagnosticar y prevenir las enfermedades, y en general sanar el cuerpo y el espíritu; además, sugiere un conjunto de contenidos teóricos y prácticos que son transmitidos de generación en generación, fundamentados en concepciones míticas y la singularidad histórico-cultural de las comunidades (12-13).

El pensamiento de los pueblos indígenas respecto a la salud está ligado a una perspectiva integral del mundo, por lo que la salud se define en términos de un bienestar integral que incorpora, infaltablemente, la dimensión espiritual, así como el bienestar biológico, psicológico, social y espiritual del individuo y de su comunidad (14).

Dentro de su forma de vida y cultura, los indígenas han desarrollado todo un sistema de prácticas y pensamientos entorno a la salud, sistemas que durante muchos ańos han sido utilizados y aún siguen vigentes en muchas comunidades (15). A este conjunto de prácticas y conocimientos se les denomina medicina tradicional o sistemas de salud indígenas, que exhiben cinco rasgos fundamentales que los definen como sistemas médicos y los posibilitan como alternativa de atención en salud para una comunidad (Tabla 1). Es gracias a esto que los pueblos indígenas, basándose en el equilibrio, la armonía y la integralidad, han logrado sobrevivir (16).

Tabla 1. Rasgos fundamentales de los sistemas médicos indígenas.

\section{CARACTERÍSTICAS}

1. Validez como etnomedicina (sistema terapéutico adaptado a contextos socioculturales y geográficos concretos que responden a las necesidades de salud de estos grupos).

2. Utilización de recursos naturales (plantas, minerales, agua, etc.), como medios terapéuticos para prevenir y combatir las enfermedades y como elementos íntimamente relacionados con la cultura y las creencias.

3. Contemplación necesaria del elemento cultural de la enfermedad. Salud/enfermedad como unidad en relación directa con el equilibrio/desequilibrio del entorno.

4. Sistemas estrechamente integrados con el resto de la cultura de la sociedad que se trate.

5. Los sistemas terapéuticos de los grupos originarios y de las culturas tradicionales forman parte de otros elementos organizativos y equilibradores del individuo, el grupo, el medio y las creencias con el cosmos.

Fuente: elaboración propia basado en Mena (2006); Mena (2005); Patińo y Sandín (2014).

Para entender el proceso de sensibilización y aprendizaje con base en la interculturalidad, es necesario aproximarse a la cosmovisión de los pueblos indígenas, pues sus conocimientos orientan los conceptos de la vida, la muerte, la salud, la enfermedad y las estrategias de curación para lograr la recuperación de la salud (17).

Un sistema médico tradicional presenta diferentes actores que están organizados jerárquicamente, donde se incluyen 
los comuneros, sobanderos, rezanderos, curanderos, parteras, los médicos tradicionales, el Jaibaná y el Chamán (el de mayor jerarquía) (12,17-18). Los Jaibanás son los médicos espirituales que requieren el don para serlo, curan los espíritus de maleficio, trabajan con plantas y animales mediante rituales. Son las personas a las que acuden inicialmente cuando presentan un problema de salud dado que es éste el que determina la causa de la enfermedad (19).

Para los indígenas, la salud es entendida como un estado de equilibrio que le permite a cada uno interactuar y convivir armónicamente con la naturaleza, consigo mismo y con los demás, encaminado al bienestar integral, a la plenitud y tranquilidad espiritual, individual y social. De este modo, la salud se expresa en las relaciones dinámicas y equilibrio entre los componentes inseparables de lo que es individual (físico, mental, emocional y espiritual) y lo que es colectivo (ecológico, político, económico, cultural, social y, una vez más, espiritual) (20).

Los indígenas tienen una concepción holística de lo que es enfermedad. Para ellos el problema de salud no es de origen solo físico y va más allá de lo orgánico; por lo tanto, las intervenciones tampoco son exclusivas para el cuerpo. La enfermedad está definida en un sentido social, como la interrupción del comportamiento social normal y la habilidad del individuo para trabajar (20).

Cabe resaltar que al interior de cada contexto sociocultural particular se construye un sistema propio de clasificación de la etiología, diagnóstico, tratamiento y pronóstico de las enfermedades según lo aprendido mediante tradición oral (15). Es así como existen diferentes técnicas de diagnóstico y pronóstico de las enfermedades: la vela, el cuy, el huevo, la orina del paciente. Otros terapeutas diagnostican y pronostican bajo el efecto de plantas alucinógenas y otros interpretando sus sueńos (20).

Los tratamientos agrupan prácticas para estabilizar las energías que producen dańo en el espíritu como rituales y plantas medicinales preparadas en ungüentos, pomadas, infusiones, bebidas, maceraciones y emplastos, cuyo éxito depende de la fe de las personas que las utilizan (6). Esta estrecha relación con la naturaleza les ha posibilitado una comprensión y conocimiento de la clasificación, composición y uso de las plantas de su hábitat respectivo enlazándose con lo mágico y religioso de la cosmovisión de los pueblos indígenas (12).
Así mismo, en el abordaje del proceso salud-enfermedad, las comunidades indígenas hacen diferencia entre sanación y curación. La sanación hace referencia al manejo físico y espiritual de la enfermedad a través de la etnobotánica (uso de las propiedades curativas de las plantas y las energías o espíritus que fluyen a través de ellas) y la intermediación del médico tradicional como agente que restablece las relaciones del hombre con el mundo espiritual. Al contrario, curar alude a un trabajo paliativo donde se aborda el componente físico sin restablecer la armonía espiritual (21).

\section{INTERCULTURALIDAD}

El interculturalismo tiene mayor alcance analítico y normativo, dado que da cuenta de un rango más amplio de fenómenos de diversidad, intenta ir más allá de la tolerancia y la coexistencia entre culturas para alcanzar el respeto y la convivencia, y defiende una igualdad y justicia sustanciales más que formales entre ellas (22).

La interculturalidad surge como un medio para redefinir las relaciones entre los Estados-nación poscoloniales y los pueblos indígenas, con el fin de diversificar la sociedad y emana como un mecanismo para reconocer la existencia de diferentes culturas. Así se pretende dar solución a las formas históricas de sometimiento y de imposición de saberes que hacían parte de siglos pasados (23), debido a los métodos utilizados para capturarlos, obligarlos a trabajar e imponer su lengua, su alimentación, su vestimenta y en general su cultura. Cabe recordar que, durante la colonización de los territorios americanos, la corona espańola impuso nuevas formas de organización, implicando un aspecto de asimetría y hegemonía, tanto en lo físico y económico, como en lo cultural y civilizatorio. Así, la potencia colonizadora no solo ocupa territorio ajeno y lo cultiva, sino que lleva e impone su propia cultura y civilización, incluyendo la lengua, religión y las leyes (24). Por tanto, la interculturalidad opera como objeto de atención académica y como principio o meta de programas políticos, educativos, epidemiológicos, administrativos o filosóficos referidos a poblaciones indígenas, afrodescendientes y migrantes, principalmente (25).

El modelo interculturalista promueve la visión de mundo tanto individual como colectiva, se abre a otras culturas y facilita la descentralización cultural, con la construcción de nuevos esquemas de pensamiento (26). La interculturalidad se asume desde el respeto, más que de la tolerancia desde las relaciones, de modo que se entiende como una teoría realmente inclusiva y no excluyente (27). Es un modelo que lleva a reconocer y 
aceptar el pluralismo cultural como una realidad social, a contribuir al desarrollo de la sociedad desde el marco de la igualdad de derechos y de la equidad, y al establecimiento de relaciones interétnicas armoniosas $(28,29)$.

De este modo, dentro de los proyectos de difusión de la interculturalidad se encuentra la etnoeducación que, sustentada en distintos saberes como los de la antropología, la lingüística y el derecho, define los rasgos que deben caracterizar la educación que reciban o adelanten por sí mismos los indígenas y los afrodescendientes $(25,30,31)$.

Para el ańo 2005, en Colombia se presupuestó que la población indígena alcanzaba el 3,43 de la población total $\mathrm{y}$, de este porcentaje, aproximadamente 37.327 personas hacen parte de la comunidad indígena emberá (3). A pesar de la importante proporción que suponen estas poblaciones para la sociedad colombiana, estos grupos étnicos son poco priorizados para muchos sectores como la salud y la educación por la lejanía de estas comunidades de las cabeceras municipales, lo que dificulta el acceso y la distribución de recursos, sumado a la poca tolerancia racial que en algunos casos se puede encontrar $(3,32)$.

\section{INTERCULTURALIDAD EN SALUD}

La interculturalidad suele confundirse con pluriculturalidad y multiculturalidad, ya que son términos que se prestan para la confusión pero que en su trasfondo son diferentes. La interculturalidad hace referencia a "entre culturas", mas no implica que convivan sin relación como sí ocurre en el caso de la pluriculturalidad, o que se presenten interacciones hegemónicas como ocurre en la multiculturalidad (8, 12-13, 33). En el enfoque de la interculturalidad prima el sujeto sobre las diferencias culturales y sociales $(13,34)$.

En concordancia, en lengua emberá se usa el término Wikira Ekni para referirse a la salud, que es el eje del equilibrio entre hombre, naturaleza, ancestros y alterego; y es un término cercano a salud intercultural por el holismo requerido en la prestación de algunos servicios de salud $(13,34)$.

En países como Colombia, México, Chile y Perú, la interculturalidad en salud constituye un pilar de las iniciativas en salud para los pueblos indígenas, donde se busca el reconocimiento del saber ancestral (34).

Los servicios de salud con enfoque intercultural incluyen saberes tradicionales en la solución de los problemas de salud, y la aceptación de representantes de la comunidad en la planeación de proyectos sanitarios, en la prevención de enfermedades, en la mejoría de la relación médico-paciente, entre otros $(13,35)$. Para implementar la interculturalidad en salud, no solo se requiere de un personal sensibilizado y capacitado en este modelo de atención, sino que además se necesitan otros elementos como programas de atención y servicios de salud basados en los requerimientos específicos de cada comunidad (Tabla 2).

Tabla 2. Elementos necesarios para implementar la interculturalidad en salud

\begin{tabular}{|c|c|}
\hline Elemento & Característica \\
\hline \multirow[b]{2}{*}{ Personal } & $\begin{array}{l}\text { Sensibilizado y capacitado } \\
\text { en el modelo de Atención } \\
\text { Intercultural }\end{array}$ \\
\hline & $\begin{array}{l}\text { Respetuoso, que reconozca y } \\
\text { comprenda los conocimientos } \\
\text { y prácticas de la población } \\
\text { en relación con el proceso } \\
\text { salud-enfermedad. }\end{array}$ \\
\hline \multirow{6}{*}{ Programa de atención } & $\begin{array}{l}\text { Validados con la población } \\
\text { organizada en y desde las } \\
\text { comunidades. }\end{array}$ \\
\hline & $\begin{array}{l}\text { Con elementos intercultu- } \\
\text { rales. }\end{array}$ \\
\hline & $\begin{array}{l}\text { Con agentes comunitarios de } \\
\text { salud. }\end{array}$ \\
\hline & $\begin{array}{l}\text { Que involucran a la población } \\
\text { en la solución de sus proble- } \\
\text { mas de salud y autocuidado. }\end{array}$ \\
\hline & $\begin{array}{l}\text { Que reconocen y favorecen } \\
\text { el desarrollo de la medicina } \\
\text { tradicional. }\end{array}$ \\
\hline & $\begin{array}{l}\text { Que desarrollan la salud } \\
\text { intercultural. }\end{array}$ \\
\hline \multirow{4}{*}{ Servicios de salud } & $\begin{array}{l}\text { Adecuados a características } \\
\text { culturales, creencias, costum- } \\
\text { bres, procedencia, etc. del } \\
\text { usuario. }\end{array}$ \\
\hline & $\begin{array}{l}\text { Con agentes comunitarios de } \\
\text { salud. }\end{array}$ \\
\hline & $\begin{array}{l}\text { Que incluyen elementos } \\
\text { interculturales. }\end{array}$ \\
\hline & $\begin{array}{l}\text { Que desarrollan la salud } \\
\text { intercultural. }\end{array}$ \\
\hline \multirow{2}{*}{ Espacios físicos } & $\begin{array}{l}\text { Que incluyen elementos } \\
\text { interculturales. }\end{array}$ \\
\hline & $\begin{array}{l}\text { Que desarrollan la salud } \\
\text { intercultural. }\end{array}$ \\
\hline
\end{tabular}


En lo que respecta a la educación médica actual, es limitada la inclusión de temas sobre interculturalidad en salud en el pensum de los programas de medicina y de las ciencias de la salud en general. En México, por ejemplo, solo el $27 \%$ de las instituciones de formación alópata incluyen temas sobre plantas medicinales y medicina tradicional indígena, el 50\% de estos es optativo y el 53\% se dicta con baja proporción de créditos dentro del programa (12).

Esta limitante impide el correcto desarrollo de la educación intercultural, dado que el personal de salud no se encuentra capacitado ni cuenta con las herramientas necesarias para dirigirse a la población indígena y así establecer una comunicación asertiva que permita el diálogo de saberes y posteriormente llevar a cabo soluciones que impacten positivamente en la situación de salud de la comunidad.

Dentro del contexto de una política de atención sanitaria intercultural, el conocimiento de la tradición indígena sobre plantas medicinales, tratamientos tradicionales y su abordaje dentro del espacio del conocimiento médicoacadémico, tiene pertinencia por las características sociodemográficas y las prácticas culturales que posibilitan una mejor atención en salud $(8,12)$. La implementación de la salud intercultural involucra no solo a las grandes agencias internacionales, sino también a entidades que llegan a las poblaciones con un discurso de buena voluntad, incluyendo el Estado (17).

\section{INTERCULTURALIDAD EN EDUCACIÓN}

En el último estudio a gran escala dado a conocer por el Instituto de Estadísticas de la UNESCO en París, en el 2017, se entregaron una serie de cifras que daban cuenta de la situación de alfabetización a nivel global en ese entonces. Según el informe, existían 750 millones de personas analfabetas en el planeta, de las cuales 32 millones pertenecían a América Latina y el Caribe, lo que representaba un $4 \%$ del total de la población del mundo (36).

Para el caso de la población indígena en América Latina, la UNESCO advierte sobre los bajos niveles de aprendizaje de los nińos de estas comunidades. En Brasil, Chile, Colombia, Ecuador, El Salvador, Guatemala, Panamá y Perú, la brecha de aprendizaje entre los estudiantes indígenas y los no indígenas superaba los 15 puntos porcentuales (36).

Según el DANE del 2018, el 13,3\% de la población indígena en Colombia es analfabeta en comparación con un $4,5 \%$ del resto de la población colombiana. Adicionalmente, el 41,8\% de los indígenas no sobrepasan la educación básica primaria y solo un $6,7 \%$ de los mismos alcanza la educación superior (37).

Aunque en los últimos ańos se han evidenciado avances significativos en cuanto a las oportunidades de acceso a la educación de las poblaciones indígenas, aún persisten las desigualdades étnicas y de género. Además de esto, subyacen las inequidades geográficas que hace que los territorios indígenas sean las áreas más desfavorecidas en lo que respecta a los indicadores educativos (38).

Adicionalmente, el acceso al sistema educativo no garantiza ni la calidad ni la pertinencia cultural del proceso de enseńanza y aprendizaje de las comunidades indígenas, por lo que el respeto y la promoción de los idiomas indígenas es un aspecto central de la educación intercultural en el marco de los derechos de los pueblos, incluidos los derechos lingüísticos y a la integridad cultural, ya que por su intermedio se transmiten la cultura, los valores y la cosmovisión indígena (38).

\section{GRUPO PIRAGUAS: UN PUENTE EN EL PROCESO DE EDUCACIÓN EN SALUD DE LAS COMUNIDADES INDÍGENAS}

El grupo Piraguas es un grupo conformado por estudiantes y docentes investigadores de la Facultad de Medicina de la Universidad Pontificia Bolivariana, que tiene como objetivo facilitar el proceso de educación en salud de las comunidades indígenas emberá del Chocó, de manera que se pueda crear un diálogo de saberes entre la medicina moderna y la medicina ancestral. Promueve la implementación de modelos de atención básica en comunidades por medio de la formación de promotores de salud indígenas en temas de autocuidado y medidas de promoción y prevención, de modo que ellos participen activamente en la resolución de sus problemáticas y, basado en los conocimientos adquiridos, puedan mejorar sus condiciones de vida y disminuir los índices de morbilidad y mortalidad dentro de la comunidad.

La razón por la que se busca ese diálogo armonioso entre ambos saberes es porque este grupo ha comprendido que la medicina moderna tiene sus inicios en la medicina ancestral, puesto que, sin argumentos científicos, ha logrado impactar positivamente en la salud de muchas comunidades a través de los ańos. Adicionalmente, comprende que el diálogo de ambas medicinas permite promover la salud en este tipo de 
comunidades, dado que se suma su experiencia, su intuición, su conexión espiritual con la naturaleza y su creatividad a la hora de buscar recursos, junto con la validez científica y los resultados de múltiples estudios experimentales desarrollados a lo largo del tiempo.

Uno de los objetivos principales del grupo Piraguas es educar en hábitos de vida saludable a la comunidad indígena. Para ello, los integrantes del grupo emprenden aventuras pedagógicas y médicas, desplazándose hacia los resguardos indígenas con el fin de analizar y comprender su estilo de vida y la relación que estos tienen consigo mismos, con los demás y con su entorno. De este modo, se plantean estrategias educativas que, por medio de una comunicación asertiva y bidireccional, permitan mejorar algunas prácticas de salud en su comunidad y orientar a las comunidades en prevención de enfermedades infecciosas y crónicas.

Al llevar a cabo las actividades educativas en las comunidades indígenas, el grupo Piraguas ha evidenciado que existen prácticas deficientes en cuanto al manejo del agua para el consumo, el de aguas residuales y el reciclaje, de modo que se plantean soluciones teniendo en cuenta sus recursos y respetando sus creencias. Así mismo, se abordan temas de nutrición, parasitosis humanas, salud sexual y reproductiva, enfermedades de transmisión sexual y primeros auxilios, con el fin de profundizar en temas que, si bien ellos ya conocen, es importante reforzar y aclarar dudas que ellos presenten. Estas actividades se realizan en castellano; sin embargo, para mejorar el proceso comunicativo se cuenta con el apoyo de un traductor indígena, que permite la resolución de dudas que surjan en el proceso educativo.

Esta experiencia adquirida en los doce ańos de trabajo le ha permitido al grupo Piraguas reflexionar sobre la importancia de abordar al ser humano desde una perspectiva holística, entendiéndolo como una unidad biológica, psíquica y social, y no como lo suele hacer en muchas ocasiones la medicina occidental, como un conjunto de órganos, sin relación entre ellos, que se pueden reparar, extraer o sustituir por otros. Además, los indígenas tienen una visión del concepto saludenfermedad enfocada desde lo espiritual, por lo que sus problemáticas las abordan siempre escuchando y llevando a cabo prácticas que busquen reestablecer el equilibrio interior, mientras que la medicina moderna se ha vuelto mecanicista y convierte el cuerpo en objeto de intervención. Por lo anterior, es fundamental acoger la concepción indígena a la medicina occidental, con el fin de que se recupere el humanismo y el enfoque integral en la atención médica y se rescaten los valores que reconozcan la dignidad de los pacientes y honren el ejercicio de la práctica médica.

En este intercambio de saberes se ha evidenciado que los indígenas muestran interés y están abiertos a establecer comunicación e intercambiar conocimientos con otras personas no pertenecientes a su comunidad, siempre y cuando éstas se acerquen de manera pacífica y estén dispuestas a escuchar y enseńar sin imponer saberes.

\section{CONTRIBUCIÓN DE LOS AUTORES}

Todos los autores de este artículo participaron en la búsqueda de información y en la redacción del manuscrito.

\section{FINANCIAMIENTO Y CONFLICTOS DE INTERESES}

Los autores no declaran conflictos de interés y declaran no haber recibido ninguna fuente de financiamiento.

\section{REFERENCIAS}

1. Bericat E. Cultura y Sociedad. En: De Ussel JI, Trinidad A, Soriano RM. La sociedad desde la sociología: una introducción a la sociología general. Vol 5. Espańa. Tecnos. 2016. p 123-152.

2. Departamento Administrativo Nacional De Estadística (DANE). Dirección de censos y demografía. 2007. Colombia una nación multicultural, su diversidad étnica. Disponible en: https://www.dane.gov.co/files/ censo2005/etnia/sys/colombia_nacion.pdf

3. Ministerio de Cultura. Caracterizaciones de los pueblos indígenas de Colombia dirección de poblaciones. Embera-Dóbida gente de río. Disponible en: https:// www.mincultura.gov.co/prensa/noticias/Documents/ Poblaciones/PUEBLO\%20EMBERA-D\%C3\%93BIDA.pdf

4. Constitución política de Colombia [Const]. Art. 7, 8 [Titulo 1] de 1991. Disponible en: http:// wsp.presidencia.gov.co/Normativa/Documents/ Constitucion-Politica-Colombia.pdf

5. Cardona-Arias JA. Sistema médico tradicional de comunidades indígenas Emberá-Chamí del Departamento de Caldas-Colombia. Revista de Salud Pública. 2012;14(4):630-643. 
6. Cardona-Arias JA, Rivera-Palomino Y. Representaciones sociales sobre medicina tradicional y enfermedades foráneas en indígenas EmberaChamí de Colombia. Revista Cubana de Salud Pública. 2012; 38(3):471-483.

7. Ministerio de Salud y Protección Social. Perfil de Salud de la Población Indígena, y medición de desigualdades en salud. Colombia 2016. Contexto actual sobre los pueblos indígenas. Disponible en: https://www.minsalud.gov.co/ sites/rid/Lists/BibliotecaDigital/RIDE/VS/ED/PSP/Perfilsalud-pueblos-indigenas-colombia-2016.pdf

8. Castillo Guzmán E, Guido Guevara SP. La interculturalidad: żprincipio o fin de la utopía?. Rev. Colomb. Educ. 2015; 0(69):17.43 https://doi. org/10.17227/01203916.69rce17.44

9. Barrera LR. El concepto de la cultura: definiciones, debates y usos sociales. Revista de Claseshistoria. 2013; 343:1-24

10. Hasen FN. Interculturalidad en salud: Competencias en prácticas de salud con población indígena. Cienc. enferm. 2012; 18(3):17-24. doi: https://doi. org/10.4067/S0717-95532012000300003

11. Maya AP, Cruz M. De eso que se ha llamado interculturalidad en salud: un enfoque reflexivo. Rev Univ Ind Santander Salud. 2018; 50(4):366-384. doi: https://doi.org/10.18273/revsal.v50n4-2018010

12. Macías R. Factores culturales y desarrollo, cultural comunitario. Reflexiones desde la práctica. Universidad de Las Tunas "Vladimir Ilich Lenin". 2011. Disponible en: https://www.eumed.net/libros-gratis/2011c/985/ index.htm

13. Aparicio AJ. La medicina tradicional como medicina ecocultural. Gazeta de Antropología. 2005; 21(10). Disponible en: http://www.ugr.es/ pwlac/G21_10Alfonso_Aparicio_ Mena.pdf

14. Departamento Administrativo Nacional de Estadísticas (DANE). (2005). Dirección de censos y demografía. La visibilización estadística de los grupos étnicos colombianos. Disponible en: https://www.dane.gov. co/files/censo2005/etnia/sys/visibilidad_estadistica_ etnicos.pdf

15. 15. Duque LF. La Interculturalidad Colombiana: Mirada Necesaria para Comprender el Territorio y Superar Conflictos. Bogotá (Colombia); 2015. Disponible en: https://repository.usta.edu.co/ bitstream/handle/11634/2200/Duqueluisa2015. pdf? sequence $=1$ \&isAllowed $=y$

16. Goicochea E. Interculturalidad en salud. UCV-Scientia. 2012; 4(1): 52-55.

17. Rosas S. La interculturalidad en salud: espacio de convergencia entre dos sistemas de conocimiento. Revista Gerencia y Políticas de Salud. 2016;
15(31):10-29. http://dx.doi.org/10.11144/Javeriana. rgyps15-31.isec

18. Hall G, Patrinos AH. Indigenous Peoples, Poverty and Human Development in Latin America: 1994-2004. Washington, DC: World Bank, 2005. Disponible en: http://documents. worldbank.org/curated/en/327561468300536074/ pdf/330200ENGLISH01Poverty1Exec1Summ1en.pdf

19. Arboleda JC, Vargas L, Fiscal L, López K, Mejía YT. Percepciones y prácticas sobre salud-enfermedad y muerte de una madre emberá chamí, una aproximación etnográfica. Cultura de los Cuidados. 2016; 23(54):3108. http://dx.doi.org/10.14198/cuid.2019.54.27

20. Organización Panamericana de la Salud. La salud de los Pueblos indígenas de las américas: Conceptos, estrategias, Prácticas y desafíos. OPS. 2009: 1-62. Disponible en: https://www.paho.org/hq/ dmdocuments/2009/53-SPI-conceptos-estrategias. PDF.pdf

21. Instituto interamericano de derechos humanos. Medicina indígena tradicional y Medicina convencional. OPS. 2006. Disponible en: https://www.academia. edu/24987343/INSTITUTO_INTERAMERICANO_DE_ DERECHOS_HUMANOS_ORGANIZACI\%C $3 \% 93 \mathrm{~N}_{-}$ PANAMERICANA_DE_LA_SALUD_medicina_ ind \% C3\%ADgena_tradicional_y_medicina_ convencional

22. Cruz E. Multiculturalismo e interculturalismo: una lectura comparada. Cuadernos Interculturales. 2013; 11(20):45-76.

23. Dietz G. Interculturalidad: una aproximación antropológica. Perfiles educativos. 2017; 39(156):192-207.

24. Eudave I. Invención, colonización y memoria indígena en la narrativa de fray bernardino de sahagún. Diálogo andino. 2016; 49: 57-72. http://dx.doi.org/10.4067/ S0719-26812016000100008

25. Patińo AE, Sandín M. Diálogo y respeto: bases para la construcción de un sistema de salud intercultural para las comunidades indígenas de Puerto Narińo, Amazonas, Colombia. Salud Colectiva. 2014; 10(3): 379-396. https://doi.org/10.18294/sc.2014.400

26. Peńalva A, Soriano E. Objetivos y contenidos sobre interculturalidad en la formación inicial de educadores y educadoras. Estudios sobre educación. 2010; 18:37-57.

27. Pineda R. La Constitución de 1991 y la perspectiva del multiculturalismo en Colombia. Alteridades. 1997; 7 (14): 107-29.

28. Pińeros M. Algunas reflexiones en torno a la salud de comunidades indígenas. Biomédica. 1994; 14:187-92. https://doi.org/10.7705/biomedica.v14i3.2103 
29. Tirzo J, Hernández JG. Relaciones interculturales, interculturalidad y multiculturalismo; teorías, conceptos, actores y referencias. Cuicuilco. 2010; 17(48):11-34.

30. Ramírez S. Salud, globalización e interculturalidad: una mirada antropológica a la situación de los pueblos indígenas de Sudamérica. Cięncia \& Saúde Coletiva. 2014;19(10): 4061-9. https://doi.org/10.1590/1413812320141910.09372014 .

31. Sánchez S. Hacia la interculturalidad desde la cultura de paz. Una perspectiva educativa. Revista De Educaçăo E Humanidades. 2011; 1:117-136. https://doi. org/10.30827/dreh.v0i1.7162

32. Vélez TI. Desplazamiento y etnicidad: fracasos del multiculturalismo en Colombia. Desacatos. 2013; 41:155-173.

33. 33. Walsh C. Interculturalidad, plurinacionalidad y decolonialidad: las insurgencias político-epistémicas de refundar el Estado. Tabula Rasa. 2008; 9:131-152.

34. Jiménez BF. Paz intercultural. Europa, buscando su identidad. Revista De Paz Y Conflictos. 2016; 9(1):1345. https://doi.org/10.30827/revpaz.v9i1.4903

35. Zubrick SR, Lawrence D, de Maio J, Biddle N. Testing the reliability of a measure of Aboriginal children's mental health: An analysis based on the Western Australian Aboriginal Child Health Survey. Canberra, Australian Bureau of Statistics. ABS Catalogue. 2006; No.1351.0.55.011.

36. Organización de las Naciones Unidas para la Educación, la Ciencia y la Cultura/Laboratorio Latinoamericano de Evaluación de la Calidad de la Educación. Factores asociados al logro cognitivo de los estudiantes de América Latina y El Caribe. 2010. Disponible en: http:// unesdoc.unesco.org/images/0018/001867/186769S.pdf

37. DANE. Población indígena de Colombia. Resultado del Censo nacional de población y vivienda. 2018. Disponible en: https://www.dane.gov.co/ files/investigaciones/boletines/grupos-etnicos/ presentacion-grupos-etnicos-2019.pdf

38. CEPAL. Los pueblos indígenas en América Latina. Avances en el último decenio y retos pendientes para la garantía de sus derechos. 2014: 99-108. Disponible en: https://repositorio.cepal.org/bitstream/ handle/11362/37050/4/S1420783_es.pdf 